\title{
The $\alpha+\mathrm{d} \rightarrow{ }^{6} \mathrm{Li}+\gamma$ reaction and the second Lithium puzzle
}

\author{
Alessandro Grassi ${ }^{1, *}$ and Laura Elisa Marcucci ${ }^{2,3}$ \\ ${ }^{1}$ M. Smoluchowski Institute of Physics, Jagiellonian University, PL-30348 Kraków, Poland \\ ${ }^{2}$ Dipartimento di Fisica “E. Fermi”, Pisa University, I-56127 Pisa, Italy \\ ${ }^{3}$ Istituto Nazionale di Fisica Nucleare, Sezione di Pisa, I-56127 Pisa, Italy
}

\begin{abstract}
A brief review on the second Lithium problem is presented. In particular the focus is on the $\alpha+\mathrm{d} \rightarrow{ }^{6} \mathrm{Li}+\gamma$ reaction and on the details of the different $\alpha$-d potential models and the theoretical approximations done during the evaluation of the astrophysical $S$-factor.
\end{abstract}

\section{Introduction}

This brief review deals with one of the currently open problems in astrophysics - the second Lithium problem. It stems from the observation published in Ref. [1] of an abnormal value of the ${ }^{6} \mathrm{Li}$ abundance with respect to the ${ }^{7} \mathrm{Li}$ abundance. The observation had been done in old halo stars, which are supposed to preserve the Big Bang Nucleosynthesis (BBN) isotopes abundances. The observed ${ }^{6} \mathrm{Li} /{ }^{7} \mathrm{Li}$ ratio was found to be approximately a thousand times bigger than the predicted one. This inconsistency triggered new interest in the study of the process $\alpha+\mathrm{d} \rightarrow{ }^{6} \mathrm{Li}+\gamma$, which is supposed to be the only reaction which produces ${ }^{6} \mathrm{Li}$ during the BBN.

Most of the studies about this reaction use cluster models to simplify the nuclear six-body problem. They consider the ${ }^{6} \mathrm{Li}$ to be a bound system of structureless $\alpha$ and d particles. Moreover phenomenological $\alpha$-d potentials are used. These are always fitted to reproduce the ${ }^{6} \mathrm{Li}$ binding energy with respect to the $\alpha$-d threshold and the $\alpha$-d scattering phase-shifts. The observable of interest in all these studies has been the astrophysical $S$-factor, defined as

$$
S(E)=E \sigma(E) \exp \sqrt{\frac{E_{G}}{E}},
$$

where $E_{G}$ is the Gamow energy, $E$ is the center-of-mass energy and $\sigma(E)$ is the capture cross section. The astrophysical $S$-factor has been evaluated by expanding the scattering wave function into multipoles and then by performing the so-called long-wavelength approximation - retaining only the contribution of the electric dipole and quadrupole moments. This is possible due to the low energies of the particles at BBN energies, which are $\simeq 50-300 \mathrm{keV}$.

\section{The five potential models}

In Ref. [2] a study of existing and new models has been done. Three existing models were taken from literature, they are

\footnotetext{
*e-mail: alessandro.grassi@doctoral.uj.edu.pl
} 
- a Wood-Saxon central potential with a $\mathbf{L} \cdot \mathbf{S}$ coupling term, from Ref. [3] $\left(V_{H}\right)$;

- a Gaussian potential, from Ref. [4] $\left(V_{T}\right)$;

- a modified version of $V_{H}$, it additionally reproduces the $S$-state asymptotic normalization coefficient (ANC), from Ref. [5]( $\left.V_{M}\right)$.

These models contain no tensor term and, therefore, are not able to reproduce the ${ }^{6} \mathrm{Li} D$-wave component. In Ref. [6] a potential model with a tensor term is constructed to reproduce the ${ }^{6} \mathrm{Li}$ bound state properties and the $J^{\pi}=1^{+}$scattering phase shifts. In Ref. [2] a new potential terms has been added in order to reproduce all the scattering phase shifts up to total angular momentum $J=3$ and orbital angular momentum $\ell=2$. The two new potentials containing a tensor term are therefore

- the Gaussian central and tensor term obtained from Ref. [6] $\left(V_{T}\right)$;

- a modified version of $V_{T}$, which additionally reproduces the ${ }^{6} \mathrm{Li}$ ANC, first published in Ref. [2] $\left(V_{G}\right)$.

The low energies of the incoming particles causes the reaction to be fairly sensitive to the asymptotic tails of the initial and final wave function. It is therefore crucial to reproduce the ${ }^{6} \mathrm{Li}$ ANC, a work well done by $V_{T}, V_{M}$ and $V_{G}$.

\section{The astrophysical S-factors}

As noticed in Sect. 1, a multipole long-wavelength approximation has always been used to study this reaction. In this approximation, the partial cross section, with respect to the initial total (orbital) angular momentum $J_{i}\left(\ell_{i}\right)$ and the order of the multipole transition $\Lambda$, can be expressed as [2]

$$
\begin{aligned}
& \sigma_{\ell_{i} J_{i}}^{(\Lambda)}(E)=\frac{8 \pi \alpha}{v_{\mathrm{rel}} k^{2}} \frac{q}{1+q / m_{6}} \frac{Z_{e}^{(\Lambda) 2}}{[(2 \Lambda+1) ! !]^{2}} \frac{(\Lambda+1)(2 \Lambda+1)}{\Lambda}\left(2 \ell_{i}+1\right)\left(2 J_{i}+1\right) \\
& \quad \times\left[\sum_{\ell_{f}}(-)^{\ell_{f}} \sqrt{2 \ell_{f}+1}\left(\begin{array}{ccc}
\ell_{f} & \Lambda & \ell_{i} \\
0 & 0 & 0
\end{array}\right)\left\{\begin{array}{ccc}
J_{i} & \ell_{i} & 1 \\
\ell_{f} & J_{f} & \Lambda
\end{array}\right\} \int \mathrm{d} r \varphi_{{ }^{\mathrm{Li}}}^{\ell_{f}}(r) f_{\Lambda}(q r) \varphi_{\alpha+d}^{\ell_{i} J_{i}}(r)\right]^{2},
\end{aligned}
$$

where $\alpha$ is the fine structure constant, $v_{r e l}(k)$ is the relative velocity (momentum) between the $\alpha$ and d particles, $q$ is the photon momentum, $m_{6}$ is the ${ }^{6} \mathrm{Li} \mathrm{mass}, \ell_{f}$ is the ${ }^{6} \mathrm{Li}$ orbital angular momentum, $\varphi_{\alpha+d}^{\ell_{i} J_{i}}(r)$ is the initial $\alpha$-d wave function and $\varphi_{6 \mathrm{Li}}^{\ell_{f}}(r)$ is the final ${ }^{6} \mathrm{Li}$ wave function. $Z_{e}^{(\Lambda)}$ is called effective charge and is defined as

$$
Z_{e}^{(\Lambda)} \equiv Z_{d}\left(\frac{m_{\alpha}}{m_{\alpha}+m_{d}}\right)^{\Lambda}+Z_{\alpha}\left(-\frac{m_{d}}{m_{\alpha}+m_{d}}\right)^{\Lambda},
$$

while the functions $f_{\Lambda}(x)$ (where $x=q r$ ) are defined as

$$
\begin{aligned}
& f_{1}(x)=3 \frac{\left[\left(x^{2}-2\right) \sin x+2 x \cos x\right]}{x^{2}}, \\
& f_{2}(x)=15 \frac{\left[\left(5 x^{2}-12\right) \sin x+\left(12-x^{2}\right) x \cos x\right]}{x^{3}} .
\end{aligned}
$$

The final $S(E)$ is then obtained as

$$
S(E)=E \exp \sqrt{\frac{E_{G}}{E}} \sum_{\ell_{i}=0}^{2} \sum_{J_{i}=0}^{3} \sum_{\Lambda=0}^{2} \sigma_{\ell_{i} J_{i}}^{(\Lambda)}(E)
$$




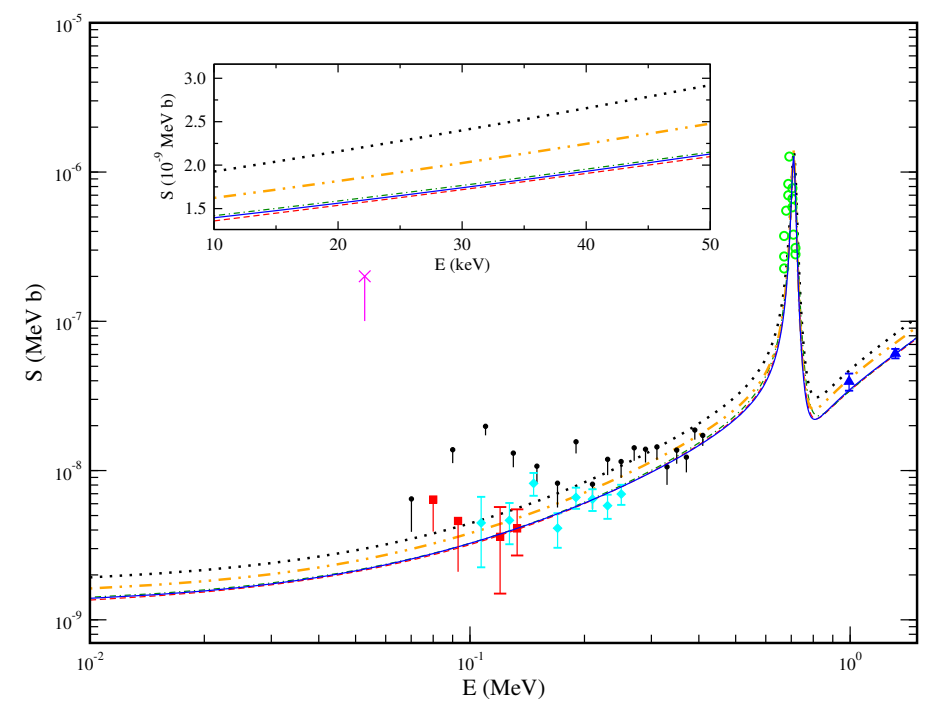

Figure 1. The total astrophysical $S$-factor, as calculated in Ref. [2], in the energy range $10 \mathrm{keV}-1.5$ $\mathrm{MeV}$. The experimental data have been taken from Ref. [7-13]. In the insert, the tail of the $S$-factor in the energy range 10-50 keV. The dotted (black), dashed (red), dot-dashed (green), dot-dot-dashed (orange) and solid (blue) lines correspond to the results obtained with the $V_{H}, V_{T}, V_{M}, V_{D}$ and $V_{G}$ potentials, respectively.

The results for the astrophysical $S$-factor for each potential are shown in Fig. 1, with the available experimental data taken from Ref. [7-13]. As it is possible to see from this figure, the three potentials which reproduce the ANC are in good agreement with each other at BBN energies, while the other two give a bigger value for the $S$-factor in this energy region. It is also interesting to notice that it is still not possible to rule out any of the five potentials, being the experimental data not accurate enough to discern about them. Finally there is no significant difference between the two potentials which reproduce the ${ }^{6} \mathrm{Li} D$-state and the other three.

\section{Conclusions}

The study of Ref. [2], here briefly reviewed, gives the most up-to-date theoretical calculations for the $\alpha+\mathrm{d} \rightarrow{ }^{6} \mathrm{Li}+\gamma$ astrophysical $S$-factor. They prove that the ${ }^{6} \mathrm{Li} D$-state contribution is negligible and that the theoretical uncertainty is at most of few $\%$, when the ${ }^{6} \mathrm{Li} \mathrm{ANC}$ is reproduced. Still there are no significant discrepancies with previous calculations when the primordial ${ }^{6} \mathrm{Li}$ abundance is predicted with these new ingredients. Thus the second Lithium problem is still a puzzle with respect to our current knowledge.

\section{References}

[1] M. Asplund, D. Lambert, P. E. Nissen, F. Primas, and V. Smith, Astrophys. J. 644, 229 (2006)

[2] A. Grassi, G. Mangano, L. E. Marcucci, O. Pisanti, Phys. Rev. C 96, 045807 (2017)

[3] F. Hammache, et al., Phys. Rev. C 82, 065803 (2010) 
[4] E. M. Tursunov, S. A. Turakulov, and P. Descouvemont, Phys. At. Nucl. 78, 193 (2015)

[5] A. M. Mukhamedzhanov, L. D. Blokhintsev, and B. F. Irgaziev, Phys. Rev. C 83, 055805 (2011)

[6] S. B. Dubovichenko, Phys. At. Nucl. 61, 162 (1998)

[7] R. G. H. Robertson, et al., Phys. Rev. Lett. 47, 1867 (1981)

[8] J. Kiener, et al., Phys. Rev. C 44, 2195 (1991)

[9] P. Mohr, et al., Phys. Rev. C 50, 1543 (1994)

[10] F. E. Cecil, J. Yan, and C. S. Galovich, Phys. Rev. C 53, 1967 (1996)

[11] S. B. Igamov and R. Yarmukhamedov, Nucl. Phys. A 673, 509 (2000)

[12] M. Anders et al., Phys. Rev. Lett. 113, 042501 (2014)

[13] D. Trezzi, et al., Astroparticle Physics 89, 57 (2017) 